Meta

Journal des traducteurs

Translators' Journal

\title{
Quand les médias traduisent la crise : les métaphores utilisées par la presse généraliste pendant la crise des subprimes
}

\section{Pier-Pascale Boulanger}

Volume 61, numéro hors-série, 2016

Sciences en traduction

Sciences in Translation

URI : https://id.erudit.org/iderudit/1038690ar

DOI : https://doi.org/10.7202/1038690ar

Aller au sommaire du numéro

Éditeur(s)

Les Presses de l’Université de Montréal

ISSN

0026-0452 (imprimé)

1492-1421 (numérique)

Découvrir la revue

Citer cet article

Boulanger, P.-P. (2016). Quand les médias traduisent la crise : les métaphores utilisées par la presse généraliste pendant la crise des subprimes. Meta, 61, 144-162. https://doi.org/10.7202/1038690ar
Résumé de l'article

Dans la couverture médiatique de la crise des prêts hypothécaires à risque (dits subprimes) qui a éclaté en août 2007, la traduction s'est trouvée mobilisée du fait que les déclencheurs de cette crise étaient d'origine américaine et ont dû être expliqués par les journalistes canadiens aux lectorats anglophones et francophones. Pour présenter avec une économie de moyens des concepts compliqués et souligner la gravité de la situation, les journalistes ont recouru aux métaphores de l'accident, du cataclysme, de la catastrophe et de l'épidémie. Après analyse des métaphores conceptuelles trouvées dans $L a$ Presse, Le Devoir, The Toronto Star et The Globe and Mail, l'article démontre que, loin d'être banales, elles confortent une idéologie néolibérale de l'économie. 


\title{
Quand les médias traduisent la crise: les métaphores utilisées par la presse généraliste pendant la crise des subprimes
}

\author{
PIER-PASCALE BOULANGER \\ Université Concordia, Montréal, Canada \\ pierpascale.boulanger@concordia.ca
}

\section{RÉSUMÉ}

Dans la couverture médiatique de la crise des prêts hypothécaires à risque (dits subprimes) qui a éclaté en août 2007 , la traduction s'est trouvée mobilisée du fait que les déclencheurs de cette crise étaient d'origine américaine et ont dû être expliqués par les journalistes canadiens aux lectorats anglophones et francophones. Pour présenter avec une économie de moyens des concepts compliqués et souligner la gravité de la situation, les journalistes ont recouru aux métaphores de l'accident, du cataclysme, de la catastrophe et de l'épidémie. Après analyse des métaphores conceptuelles trouvées dans $L a$ Presse, Le Devoir, The Toronto Star et The Globe and Mail, l'article démontre que, loin d'être banales, elles confortent une idéologie néolibérale de l'économie.

\begin{abstract}
In the media coverage of the subprime crisis of August 2007, it is through translation that journalists worked to explain its causes, which were of American origin, to the Canadian French and English readerships. In order to efficiently convey complicated concepts and the gravity of the situation, journalists resorted to metaphors of the accident, the cataclysm, the catastrophe and the epidemy. The article analyzes the conceptual metaphors found in La Presse, Le Devoir, The Toronto Star and The Globe and Mail and argues that far from being harmless commonplaces they propagate a neoliberal ideology of economics.
\end{abstract}

\section{MOTS-CLÉS/KEYWORDS}

analyse critique des métaphores, métaphores conceptuelles de la crise financière, traduction des nouvelles, presse canadienne, crise des prêts hypothécaires à risque critical metaphor analysis, conceptual metaphors of the financial crisis, news translation, Canadian press, subprime mortgage crisis

Like Botox to smooth out wrinkles, central banks can inject liquidity to smooth over financial markets. And like Botox, these injections don't make permanent changes - the financial injections only last for a day.

(Scoffield 2007)

\section{Introduction}

Glanée dans l'un des innombrables articles de journaux visant à expliquer la crise de liquidité survenue sur les marchés financiers en août 2007, la citation ci-dessus, riche à souhait, annonce l'objet du présent article: les métaphores du discours financier dans la presse généraliste en temps de crise. Dans cet exergue, l'analogie cosmétique dénonce les solutions transitoires qu'appliquent les gouvernements pour prévenir 
l'assèchement du crédit et la contraction économique subséquente. À bien analyser la figure de style, nous comprenons que l'injection de liquidité est au système bancaire ce que l'injection de Botox est à la peau, en cela que toutes deux produisent des effets éphémères, critiqués par la journaliste. Le corollaire qui en découle est la nécessité de trouver une solution durable qui, d'un côté, nivellerait les pics et les creux des marchés monétaires et, de l'autre, empêcherait les rides de se former sur la peau. Si cette deuxième prémisse reste informulée, elle ne demeure pas moins suggérée par la logique interne de l'argument exprimé par la journaliste, qui espérait ainsi marquer l'esprit de son lectorat.

La métaphore participe à rendre les nouvelles accrocheuses, mais elle permet aussi de vulgariser, donc de présenter avec une économie de moyens des concepts compliqués, tels que les événements survenus sur les marchés durant la crise des prêts hypothécaires à risque américains, dite crise des subprimes ${ }^{1}$. Outil rhétorique et conceptuel puissant, la métaphore mobilise des stéréotypes logico-discursifs qui contribuent à façonner l'idée que la société se fait des marchés financiers, mais aussi de l'argent en général (Nünning 2015: 47-68). Nous observerons comment le cadrage s'est effectué par les métaphores ayant servi à expliquer la crise financière, dans un corpus d'articles de quatre journaux canadiens parus en août et en septembre 2007. Durant la crise de liquidité déclenchée par les prêts hypothécaires subprimes - ou, par télescopage, "crise des subprimes"-, les journalistes canadiens ont, tant en anglais qu'en français, mobilisé des métaphores qui ont donné à voir la crise comme étant imprévisible. Avant de présenter celles-ci, nous traiterons de la métaphore dans la langue de spécialité de l'économie et dans le journalisme financier.

Nombre de travaux ont été menés depuis les années 1980 sur l'impossible transparence du discours médiatique et la manipulation de l'opinion publique par les médias, au nombre desquels l'essai culte Manufacturing Consent de Chomsky. Plus récemment, le travail d'analyse de Patrick Charaudeau (2005/2011) sur les médias et l'information a mis en cause l'hypothèse de la manipulation, soulignant que les organes d'information sont eux aussi manipulés du fait qu'ils interagissent avec d'autres agents dans la production des nouvelles. Les études médiatiques, l'analyse $\mathrm{du}$ discours et la linguistique cognitive qui s'intéresse au cadrage (framing) ont examiné sous des angles différents le fonctionnement du discours d'information. Dans la création de l'actualité à laquelle les médias s'affairent, les journalistes ne sont pas des relais neutres et rationnels, du fait qu'ils cadrent les événements, produisent et propagent un discours dans la sphère publique et reproduisent des idéologies préformulées (Van Dijk 1988; 1998). Celles-ci participent à construire les représentations de l'ordre social (Fowler, Hodge, et al. 1979; Van Dijk 1988; 1995; Bell 1991; 1998; Fairclough 1992; 1995). En tant qu'assemblage de significations, de métaphores, de représentations, d'images, de récits et d'affirmations, le discours permet de construire une version particulière d'un événement (Burr 1995; Dirks 2006; Li $2009 ; 2010)$. Il arrive que les journalistes inventent la réalité, comme ils l'ont fait avec les termes nouvelle économie (Maris 2002: 119) et nouvelle ère économique (Shiller 2000/2015 : 120-162). Par ailleurs, une grande part de la construction des nouvelles passe par la traduction, activité fondamentale à l'ère de la mondialisation (Orengo 2005; Gambier 2006; Valdeón 2008; Bielsa et Bassnett 2009; Conway 2010; Van Doorslaer 2010). En visant à maximiser la réception de l'information auprès d'un destinataire d'une langue-culture autre que celui qui était visé lors de la production 
du texte d'information, la traduction se trouve pleinement engagée dans l'acte d'informer.

S’appuyant sur le présupposé de l'analyse critique du discours selon lequel diverses stratégies linguistiques servent à véhiculer un ensemble de croyances qu'un groupe social a intérêt à protéger pour maintenir un ordre des choses qui lui est profitable, notre hypothèse de travail veut que l'étude des métaphores permette de découvrir les concepts à partir desquels certaines questions politiques et économiques sont cadrées et motivées (Lakoff 2004; Rojo López et Orts Llopis 2010). Le cadre conceptuel de notre analyse prend appui sur l'impossibilité de la transparence du discours médiatique, axiome au cour de l'ouvrage phare de Patrick Charaudeau (2005/2011), selon lequel la lutte que se livrent divers organes d'information pour leur survie économique place ceux-ci sous «la houlette de la double finalité de crédibilité et de captation» (Charaudeau 2005/2011: 104).

Ainsi, le contrat d'information médiatique est, dans son fondement, marqué au sceau d'une contradiction: finalité de faire savoir qui devrait tendre vers un degré zéro de mise en spectacle de l'information et satisfaire au principe de sérieux en produisant des effets de crédibilité; finalité de faire ressentir qui devrait tendre vers des choix stratégiques de mise en scène de l'information et satisfaire au principe d'émotion en produisant des effets de dramatisation (Charaudeau 2005/2011: 74).

Le recours à la métaphore par les journalistes financiers est très fréquent, du fait que celle-ci leur permet très efficacement de susciter l'intérêt et de toucher l'affect de leur destinataire, et ainsi d'être lus par le plus grand nombre de personnes possible sur un marché de libre concurrence. Les médias visent donc autant à informer qu'à séduire, mais les effets d'information et de séduction qu'ils visent à produire sur leurs lecteurs ne concordent pas nécessairement avec les effets réellement produits. «Selon le contexte dans lequel elle apparaît, une information peut produire un effet de banalisation, de saturation, d'amalgame ou au contraire de dramatisation» (Charaudeau 2005/2011: 45). Si la réception des textes demeure un problème épineux, à l'égard duquel il faut montrer la plus grande prudence, il est toutefois possible d'inférer des effets de discours. Il est admis en analyse du discours que le lexique et la fréquence de certains termes sont révélateurs des valeurs d'un groupe social (Fowler 1985), de sorte que la transformation de ces derniers soit porteuse de sens. Par exemple, lorsqu'une étude sur les occurrences de l'euphémisme subprime loan dans les journaux révèle qu'il est plus fréquent que son synonyme imagé predatory loan (Longobardi 2009), il est raisonnable d'en déduire un effet de neutralisation du discours.

Dans la crise des prêts hypothécaires subprimes qui nous intéresse, la traduction s'est trouvée mobilisée du fait que les produits financiers déclencheurs de la crise étaient d'origine américaine et ont dû être traduits, représentés, contextualisés, cadrés et expliqués par les journalistes canadiens en anglais et en français. Les concepts métaphoriques utilisés dans les journaux en temps de crise se présentent comme des indicateurs à partir desquels il devient possible de comparer la manière dont les médias anglophones et francophones représentent l'économie, l'information économique étant l'«espace où l'on parle de tout ce qui touche au pouvoir dans la vie sociale» (Charaudeau 2005/2011: 117). Pour le dire autrement, quels sont les cadres d'intelligibilité construits par les discours médiatiques et qui s'imposent dans l'espace public?

L'étude de la métaphore dans le discours financier journalistique soulève d'emblée la question de la rhétorique, au nombre des modes et stratégies d'organisation 
du discours qu'utilise le journaliste pour informer, être crédible et capter l'attention du plus grand nombre de lecteurs. Bien qu'elle s'appuie sur le savoir bimillénaire de la rhétorique, porté par La rhétorique d'Aristote et développé dans L'Institution oratoire de Quintilien, l'attention accordée à la structuration du discours financier dans les journaux est récente et directement liée au regard critique qu'ont suscité les deux crises financières survenues dans la première décennie des années 2000. Effectivement, les chercheurs sont de plus en plus nombreux à s'intéresser aux métaphores dans les textes spécialisés d'économie et de finance, afin de comprendre les motivations et les circonstances qui font naître cette figure de style, notamment en temps de crise tel qu'après l'éclatement de la bulle technologique en 2000 et de la bulle immobilière en 2007.

Moyen efficace pour exprimer des concepts abstraits à l'aide de termes concrets, la métaphore abonde en langue spécialisée. Elle sert à accomplir une certaine «transparence terminologique», laquelle permet d'éviter la création d'un néologisme, et elle offre une économie de moyens par l'usage de lexèmes d'un domaine source connu de l'interlocuteur pour lui faire comprendre un concept abstrait de manière concise (Gotti 2008: 57). La métaphore, «le plus important de tous les tropes, et l'une des plus considérables de toutes les figures, aussi bien dans l'histoire que dans la pratique actuelle» (Molinié 1992: 213), participe à construire des lieux où se réunissent des idées, qui s'installent en conceptions et finissent par faire consensus. La linguistique cognitive a montré la nature fondamentale et systématique de la métaphore dans la compréhension du monde et la cohésion culturelle, ainsi que son influence sur notre manière de parler et d'agir (Lakoff et Johnson 1980).

\section{L'économie, une science riche en métaphores}

Le discours savant de l'économie abonde en métaphores, lesquelles sous-tendent son activité scientifique en grande partie fondée sur la modélisation. Tout aussi importante que le vocabulaire dans les langues de spécialité, la métaphore a des fonctions pédagogiques et heuristiques qui dépassent la simple visée ornementale (McCloskey 1983: 503) ou rhétorique qu'on lui attribue souvent. De fait, elle permet aux sciences de se dire, de se produire et de se reproduire (Klamer et Leonard 1994; Gotti 2008: 59, 39; Vasiloaia, Gaisoa-Issa, et al. 2011 : 237). Elle «joue un rôle essentiel dans une discipline, qu'il s'agisse de l'élaboration de la théorie, de sa valorisation, de sa diffusion, de la formation de futurs chercheurs, donc de l'enseignement» (Resche 2006: 21). L'une des métaphores conceptuelles et constitutives de l'économie est la «main invisible» d'Adam $S m$ ith $^{2}$, qui, selon l'interprétation qu'en font les tenants du libéralisme économique, cadre bien avec les politiques d'autorégulation des marchés, donc de la non-intervention de l'État. La langue économique est profondément métaphorisée, de sorte que les métaphores conceptuelles participent, tout comme les catachrèses du langage courant (Lakoff et Johnson 1999), à construire les réalités sociales et culturelles. À cet égard, mentionnons le concept métaphorique du liquide ou de l'eau pour exprimer les états et les mouvements de l'argent: cash flow, income stream, liquidity, illiquidity, to liquidate, watered stocks, floating (debt; exchange rate), to inject money, to bail out, injection, flux monétaire, liquidité, illiquidité, liquider, assèchement du crédit, verser, versement, éponger ou essuyer une dette, dilution des actions, dette ou monnaie flottante, etc. Les termes nous viendraient-ils de la 
machine que l'économiste et ingénieur néozélandais Bill Phillips a inventée en 1949 en guise de modèle macroéconomique pour démontrer l'équilibre monétaire (Farber 2012, n.p.) ? Son appareil faisait circuler de l'eau, représentant de l'argent dans des tubes et des compartiments correspondant au gouvernement, au fisc, à la banque centrale, à la Monnaie, au marché financier, au marché de la consommation, aux importations et aux exportations ${ }^{3}$. Cependant, comme nous le rappellent Vandaele et Lubin dans le domaine des sciences dites exactes, "[1]a conceptualisation métaphorique, si elle se révèle extrêmement productive sur le plan de l'évolution des connaissances, peut aussi se révéler un frein à la pensée, si les projections opérant à un niveau plus ou moins conscient ne sont pas dépassées» (2005: 427).

La légitimité scientifique de l'économie suscite un débat épistémologique enflammé. En tant que science formelle, unifiée par l'hypothèse de la théorie néoclassique visant l'efficience des marchés et la rationalité des acteurs, l'économie, comme les autres sciences, légitime son statut scientifique par «l'exigence de cohérence logique, l'appel aux faits, l'évaluation par les pairs ou les experts» (Lallement 2002: 9), ainsi que par l'usage d'une langue de spécialité au demeurant «lestée» de métaphores, comme le souligne McCloskey (1983: 502), pour qui les modélisations (par exemple, une courbe) et le raisonnement mathématique sont résolument métaphoriques. Les uns la déclarent pseudoscience qui calque la physique et se drape dans un formalisme mathématique complexe (Maurin 2002; Maris 2002: 119). Les autres lui reprochent l'artificialité théorique qu'elle entretient par l'imperturbable clause ceteris paribus sic stantibus (toutes choses étant égales par ailleurs), afin d'ériger ses modélisations sans devoir tenir compte des comportements humains imprévisibles, évacués comme des «anomalies». Ces critiques sont tout d'abord imputables à l'instrumentalisation de l'économie. Effectivement, lorsqu'elle sert les politiques monétaires de l'État, l'économie poursuit une visée normative (Maris 2002: 113) qui la place au rang des sciences de la gestion. Elles sont ensuite liées à l'apport du béhaviorisme à la finance comportementale ${ }^{4}$, ébranlant ainsi l'hypothèse des marchés efficients.

Qu'en est-il de la finance? Discipline connexe à l'économie, elle applique des techniques visant l'efficacité dans l'allocation des ressources monétaires et la gestion des actifs et du risque. Son but avoué est l'amélioration du niveau de vie et la richesse. Il serait tentant de placer la finance du côté de la pratique, ancrée dans le monde du commerce et des affaires, et l'économie du côté de la théorie, qui produit son savoir dans la sphère universitaire (Partington 1995: 2). Or ce schéma réducteur ne tiendrait pas compte de la finance mathématique, qui recourt elle aussi aux modélisations et à la théorisation.

Dans le discours savant et dans le texte journalistique, les métaphores varient et n'ont pas la même fonction. Lorsque le journaliste soucieux de capter l'attention du lectorat effectue une «vulgarisation dramatisée» (Charaudeau 2005/2011: 48), s'entrecroisent les métaphores lexicalisées, donc constitutives de la langue de spécialité, et les métaphores vives de la langue journalistique. Le discours informatif, à l'instar du discours didactique à visée vulgarisatrice, vise l'accessibilité de l'information: les deux «ont affaire à des cibles larges, non spécialisées, et n'ont donc pas à fonder une vérité, mais seulement à la mettre en évidence dans un cadre d'intelligibilité accessible à un grand nombre» (Charaudeau 2005/2011: 47). Les métaphores de l'économie et de la finance ont été créées à partir de divers domaines sources. En voici un florilège ${ }^{5}$ : 
TABLEAU 1

Métaphores conceptuelles de l'économie et de la finance

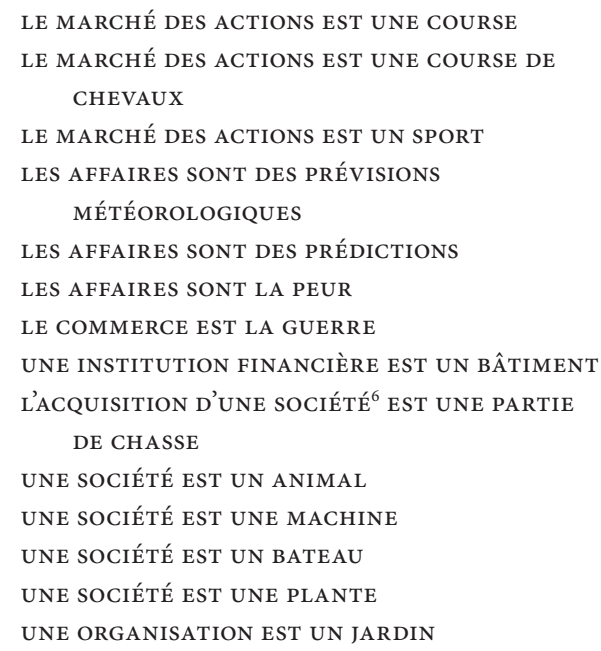

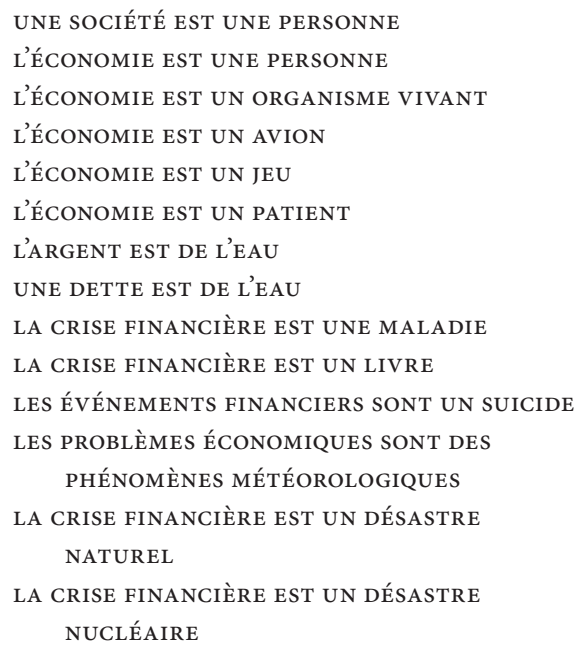

Les métaphores qui traitent de la crise en mobilisant les concepts métaphoriques du désastre naturel ou nucléaire et de la maladie nous intéressent particulièrement, car elles montrent les cadres cognitifs qui ont servi à rendre intelligibles les crises financières ${ }^{7}$. Nous reprendrons ces métaphores dans notre étude et verrons que les cadres cognitifs diffèrent chez les quotidiens anglophones et francophones.

\section{Le journalisme financier}

Dans les journaux généralistes, l'actualité financière se voit consacrer une place quotidienne dans le « rubriquage du monde social» qu'effectuent les journaux (Charaudeau 2005/2011 : 117). Depuis les années 1980, les sociétés exploitant des activités dans les domaines de la finance, de l'assurance et de l'immobilier, réunies sous l'acronyme FIRE (Finance, Insurance, Real Estate), occupent une place de plus en plus grande dans l'économie mondiale (Posca 2013: n.p.), de sorte qu'on en vient à parler d'économie financière. L'essor de la financiarisation de l'économie se répercute de manière considérable sur la société et la culture: l'optimisation des ressources, la gestion du risque et le rendement se sont imposés au fonctionnement des hôpitaux, des écoles, de la recherche et de la production culturelle (Haiven et Berland 2014: 8-9). Aussi la langue du secteur financier s'est-elle infiltrée dans le discours quotidien, notamment par les médias (Catalano et Waugh 2013: 32). Certes, les médias entretiennent une relation étroite avec la finance depuis le XVIII siècle. Par contre, la performance médiatique et surtout télévisuelle de la finance et de l'argent, devenus sujets de divertissement incarnés par des personnalités issues d'institutions financières prospères et puissantes, est assez récente (Clark, Thrift, et al. 2004: 290 et 293).

La finance occupe un «espace supraterritorial» (Bielsa et Bassnett 2009: 18) sans frontières physiques, lequel a été créé par la déréglementation des marchés et les avancées technologiques des télécommunications (Castells 2000 : 96) qui facilitent le libre investissement des capitaux à l'étranger. Dans ce «supraterritoire» (Bielsa et 
Bassnett 2009: 19), les journalistes financiers agissent en importateurs et médiateurs culturels. Lorsqu'ils couvrent des événements ou des produits financiers étrangers, qu'il s'agisse d'expliquer les divers types de prêts subprimes, la législation américaine ou le fonctionnement des marchés, ils engagent un processus de traduction complexe parce qu'à la fois interlinguistique et intralinguistique. D'une part, présenter aux membres d'une culture les événements qui se déroulent aux États-Unis et ailleurs dans le monde exige de traduire des termes que les anglophones et francophones canadiens comprendront. D’autre part, vulgariser des éléments techniques du domaine financier pour le public requiert un travail de paraphrase, donc de traduction intralinguistique. La vulgarisation motive l'invention de métaphores, notamment par l'usage de comparaisons elliptiques, qui ont la vertu de donner en peu de mots une idée générale d'un concept abstrait ${ }^{8}$.

Le journaliste écrit-traduit-adapte son texte à partir d'autres textes d'information selon un processus nommé la «transédition» par les traductologues, qui ont mis au jour l'omniprésence de la traduction dans les salles de presse. Que le journaliste travaille à traduire et à adapter le contenu d'une dépêche provenant d'une agence de presse ou qu'il réorganise le contenu tiré de diverses sources en ligne, son texte résulte d'une chaîne de manipulations. Loin d'être neutres, les nouvelles sont adaptées à un lectorat cible par diverses opérations: le filtrage de renseignements, la mise en valeur de certains acteurs et parties prenantes plutôt que d'autres (Lischinsky 2011), ainsi que la réorganisation, la suppression, l'ajout, la substitution (Gambier 2006), la généralisation et l'explicitation de certaines données (Bani 2006). Dans la confection des nouvelles, les journalistes sont souvent appelés à traduire des communiqués de presse, dont ils choisissent de reprendre le contenu sans le remettre en question (Davies 2008) ou de se distancier en adoptant un point de vue critique. Quant à ces communiqués, leur visée autopromotionnelle est largement admise (Davies 2008; Pander Maat 2007; Jacobs 1999): les sociétés commerciales les utilisent effectivement pour se montrer solides auprès des actionnaires et des analystes financiers (Davis 2002: 72), dont l'opinion positive ou négative poussera le cours des titres d'une société à la hausse ou à la baisse.

\subsection{L'interaction entre les journalistes et le milieu de la finance}

Les journalistes financiers ont fait l'objet de nombreuses critiques après la crise de 2007. La plus importante d'entre elles: ne pas avoir prévenu le public qu'une crise financière se tramait, notamment par le journalisme d'enquête. Une étude portant sur un corpus de 727 articles publiés de janvier 2000 à juin 2007 dans la presse spécialisée américaine (Starkman 2009a/2009b) révèle que des articles de fond et des opinions critiques quant aux prêts hypothécaires subprimes et à leur financement par Wall Street avaient paru entre 2000 et 2003. Après ces années, les articles traitaient plus généralement de produits d'investissement ou encore des institutions et des personnages influents de la finance. Ironiquement, c'est à cette même période que débutaient les très lucratives opérations de titrisation ${ }^{9}$ des subprimes, celles-là mêmes qui ont causé la crise d'août 2007.

On reproche au journaliste financier de ne pas aller au fond des choses (Davis 2005), bien qu'il soit enclin à mettre en lumière les bourdes des dirigeants et le sousrendement des entreprises (Doyle 2006: 433). Il est également critiqué pour son 
incapacité à analyser en profondeur les données des marchés à mesure que les produits financiers se complexifient (Tambini 2010: 159), ainsi que pour son manque de recul par rapport aux données que les sociétés lui fournissent. À ce chapitre, la partialité des données fournies par les relations publiques des sociétés est un secret de Polichinelle, si bien que celles-ci gagneraient à être systématiquement contre-vérifiées à la lumière des rapports comptables d'État (Smithers 2013: 23). Or le journaliste financier possède rarement les connaissances spécialisées qui lui permettraient de produire des données de manière autonome (Davis 2002: 70) et en temps opportun. Dans le cas de la presse financière spécialisée, il est largement reconnu que les journaux ont vingt-quatre heures de retard sur le monde réel (Davis 2005: 309; 2006: 607). De surcroît, le journaliste reconnaît que parfois il ne sait pas où trouver ses renseignements et que les analystes des sociétés ne l'informent pas toujours clairement, notamment parce que ces derniers craignent d'être cités hors contexte (Canadian Journalism Foundation [CJF] 2011: n.p.). Lorsqu'il a l'occasion de produire un dossier d'enquête, sur lequel il peut passer jusqu'à quatre mois, le journaliste accorde trop peu de temps aux sociétés visées pour qu'elles réagissent aux constats posés, et l'article paraît sans offrir le point de vue de celles-ci. Cette manœuvre s'expliquerait par le fait que le journaliste tente de faire un scoop (CJF 2011 : n.p.). Par ailleurs, la tendance à vouloir produire des nouvelles percutantes pousse le journaliste à communiquer avec plusieurs experts jusqu'à ce qu'il en trouve un qui veuille bien corroborer son point de vue (Shiller 2000/2015: 103).

Le journaliste financier veut être pris au sérieux. Pour être cru, encore faut-il qu'il soit lu, d'où la nécessité d'attirer l'attention du lecteur, notamment par un titre accrocheur, et de le maintenir intéressé par le recours à des formules et à des métaphores percutantes. À la différence du lecteur spécialisé dont l'intérêt principal est la finance et qui s'informe par la presse spécialisée qui lui est destinée (Business Week, The Wall Street Journal, The Financial Times, New York Times), le lecteur de la presse généraliste arrive aux informations financières après avoir effeuillé le cahier principal du journal, sauf lorsque la finance fait la une. L'information financière n'ayant pas préséance dans le "rubriquage» de la presse généraliste, le journaliste cherche d'autant plus à retenir l'attention de son lecteur en rendant son texte intéressant. Le fardeau de l'ennui qui pèse sur les informations financières a été soulevé à la table ronde de l'Association canadienne des journalistes sur l'état du journalisme financier (CJF 2011). Aussi les participants ont-ils évoqué le recours aux métaphores du sport et du combat comme moyen de renouveler et de pimenter les nouvelles du marché, qui, au fond, fait toujours la même chose: il monte ou il baisse ${ }^{10}$. La complexité de la finance rend la simplification par la métaphore d'autant plus attrayante aux yeux des lecteurs et des journalistes aussi, à qui on peut reprocher une certaine paresse intellectuelle, sinon un manque de curiosité (Chalmers 2012: n.p.). Efficace tant sur le plan conceptuel que psychologique, la métaphore transmet une force émotive en raison de sa proximité avec l'expérience vécue (Ortony 2001: 16) et que partage une communauté culturelle (Lakoff et Johnson 1980).

La persuasion et la séduction appellent chacune des stratégies discursives bien précises. D’une part, les données chiffrées servent à conférer une légitimation au discours financier journalistique. Les données statistiques et les citations d'experts convainquent le lecteur que ce qu'il lit est crédible. D’autre part, la métaphore permet de représenter schématiquement des réalités complexes, ce qui séduit le lecteur en 
créant chez lui l'impression gratifiante qu'il a saisi l'objet présenté et, conséquemment, établit sa confiance à l'égard du texte. Dans le cas particulier de notre corpus d'articles parus en temps de crise, les métaphores servent à expliquer les marchés financiers, souvent incompréhensibles pour les non-initiés, ainsi que la gravité de la situation. Percutantes par leur puissance de raccourci et leur force de suggestion, elles sont nombreuses et semblent suivre la prescription des rhétoriciens, soit paraître «naturelles, c'est-à-dire point tirées de trop loin, et donc raisonnablement compréhensibles» (Molinié 1992: 216). Nous verrons que cet effet du naturel joue un rôle politique primordial dans l'idéologie financière.

\section{4. Étude de corpus : les métaphores de la crise dans quatre quotidiens montréalais et torontois}

We are in the midst of a once in a century credit tsunami.

$(\text { Greenspan 2008) })^{11}$

Nous avons observé les métaphores utilisées par les journalistes financiers au moment où a éclaté la crise des prêts hypothécaires à risque. Nous exposerons les concepts métaphoriques qui ont servi à expliquer et à exprimer à un lectorat non spécialisé les causes et les effets de ce qui a en fait déclenché une crise de liquidité. Au regard d'un objet discursif aussi riche - où s'entremêlent le devoir de narrer, la responsabilité de rassurer, la nécessité de vulgariser et l'urgence de dramatiser - la question de la traduction se pose diversement. Du point de vue de la médiation culturelle, comment les réalités et produits financiers d'origine américaine, mais qui ont eu une incidence sur les marchés à l'échelle mondiale, ont-ils été présentés aux lectorats anglophones et francophones du Canada?

\subsection{Le terme subprime: définition et contexte}

Traduit le plus souvent par prêt hypothécaire à risque ou prêt hypothécaire à haut risque, le terme subprime ou subprime loan désigne un prêt qui est consenti aux débiteurs moins nantis, en situation de précarité professionnelle ou dont la cote de crédit est entachée d'un antécédent d'insolvabilité. L'adjectif subprime qualifie le taux dont ce type de prêt hypothécaire est assorti et qui a la caractéristique d'être majoré par la banque afin de couvrir le risque de défaut de paiement. Le terme se trouve en rapport d'antonymie avec l'adjectif prime, qui réfère au taux d'intérêt préférentiel, ou de premier rang, accordé à l'emprunteur solvable. C'est en fait l'idée de la majoration du taux hypothécaire qui a motivé certains à traduire subprime de manière erronée par surprime. Aux États-Unis, toute une gamme de produits à taux référencé ou variable relevant des prêts à risque était proposée aux consommateurs par les courtiers en prêts hypothécaires. Par exemple, le prêt Pick-a-Pay, à l'instar de la marge de crédit hypothécaire, laissait à la discrétion (et à l'indiscipline) de l'emprunteur le montant du remboursement mensuel. Les prêts $2 / 28$ et $3 / 27$, quant à eux, proposaient des taux très bas les deux ou trois premières années pour appâter les clients, qui, au moment de reconduire leur prêt sur les 28 ou 27 années restantes, se sont retrouvés aux prises avec un taux pleinement indexé à un taux de référence ${ }^{12}$ plus élevé et des frais de renégociation ruineux. À cette offre de produits risqués sont venues s'ajouter 
les hausses successives du taux directeur de la banque centrale américaine (la Fed), lequel a augmenté de $4 \%$ entre l'été 2003 et l'été 2007 pour calmer la surchauffe économique. Conséquemment, les emprunteurs précaires ayant souscrit un prêt hypothécaire à risque n'ont pu honorer leur traite mensuelle. Bon nombre sont tombés en défaut de paiement et ont vu leur bien immobilier saisi par la banque. Ce phénomène s'est aggravé en 2007.

Quant à la bulle immobilière américaine, les raisons qui expliquent son gonflement ainsi que l'essor fulgurant de l'activité de courtage hypothécaire depuis le début des années 2000 sont nombreuses, mais nous les résumerons aux suivantes: le faible taux directeur de la Fed motivé par la politique d'accès à la propriété sous les gouvernements de Clinton et de Bush; l'abondance de liquidités sur le marché injectée par différentes banques centrales dans le monde venues en aide au marché américain après les attentats du 11 septembre 2001 et par des fonds spéculatifs européens en quête de rendement; l'achat des créances hypothécaires par les agences de refinancement paragouvernementales ${ }^{13}$ afin de permettre aux institutions financières de libérer des fonds propres et de consentir d'autres prêts; et l'activité de titrisation très lucrative pour les banques d'investissement de Wall Street qui transformaient les créances hypothécaires en titres financiers pour les vendre à des investisseurs institutionnels. Ajoutons à ces circonstances le relâchement généralisé des conditions de prêt et les pratiques véreuses de certains courtiers hypothécaires qui ont ciblé des emprunteurs d'emblée insolvables en leur faisant souscrire des prêts de qualité douteuse tels que les prêts avec peu ou pas de preuve de solvabilité ${ }^{14}$. Aux quatre coins des États-Unis, l'incitation à faire souscrire des prêts à risque assortis d'un taux d'intérêt élevé tenait au fait que, une fois agrégées en portefeuilles puis vendues, les créances hypothécaires procuraient de généreux honoraires aux banques d'investissement qui s'occupaient de transformer les dettes hypothécaires en titres obligataires et promettaient des revenus élevés aux investisseurs institutionnels. Cependant, les défauts de paiement généralisés des emprunteurs à risque en amont ont entraîné, en aval, une forte dépréciation des titres qui étaient adossés à leurs traites mensuelles impayées.

À son apogée en 2007, la "machine à hypothèques $»^{15}$ (Federal Crisis Inquiry Commission [FCIC] $2011: 5,102,111,156)$ bat son plein et le marché des titres adossés à des créances hypothécaires frise les 1250 milliards de dollars (FCIC 2011 : 251). Les pertes à venir seront colossales, tant chez les grandes banques d'investissement que chez les investisseurs institutionnels et privés. Mais les premières et principales victimes seront les ménages américains tombés en défaut de paiement et expulsés de leur logement repris par la banque. Dans tous les registres de langue, ces subprimes ont été qualifiés d'actifs toxiques. Notre travail commence ici: comment les journalistes ont-ils métaphorisé la crise des subprimes?

\subsection{Méthodologie}

Le corpus étudié est composé des articles publiés par La Presse (Montréal), Le Devoir (Montréal), le Toronto Star et The Globe and Mail (Toronto) en août et en septembre 2007, mois durant lesquels est survenu l'événement déclencheur de la crise des subprimes. Le 9 août, le groupe BNP Paribas annonce à ses investisseurs qu'il les empêche de retirer leurs placements de trois de ses fonds spéculatifs ${ }^{16}$. Ceux-ci étant fortement 
composés de titres adossés aux subprimes dont la valeur a chuté, le groupe dit ne plus savoir combien ils valent. Cette annonce officielle sème la panique sur les marchés bancaire et boursier. La gravité de la situation est telle que les banques centrales du monde se mobilisent pour introduire des milliards ${ }^{17}$ dans le circuit interbancaire sous forme de prêts aux banques commerciales. Une étude de fréquence du terme subprime dans quatre journaux publiés durant l'année 2007 indique clairement que le mois d'août marque l'apparition du terme.

À la fois quantitative et qualitative, notre étude s'appuie sur l'analyse critique des métaphores, qui emprunte à la méthode de la linguistique computationnelle (voir Charteris-Black 2004; Partington 2006) et utilise le concordancier du logiciel WordSmith Tools 6 (Scott 2015). Il s'est agi de construire un corpus de textes représentatifs, composés des articles parus en temps de crise dans les quatre quotidiens, mais filtrés par mots-clés afin de circonscrire les textes au domaine de la finance. Notre corpus est en fait constitué de deux sous-corpus: 1) The Globe and Mail et le Toronto Star; 2) Le Devoir et La Presse. Nous avons vérifié la fréquence ${ }^{18}$ des «indices de conceptualisation métaphorique» (Vandaele et Lubin 2005), qui nous semblaient saillants par leur fréquence et la cohérence des réseaux lexicaux qu'ils forment (Vandaele, Boudreau, et al. 2006: 77), notamment ceux de l'épidémie, de l'accident, de la catastrophe et du cataclysme. Une des difficultés qui ont été soulevées dans l'analyse du discours assistée par ordinateur tient au choix et au classement que le chercheur fait des indices (Stefanowitsch 2006b). Par exemple, est-il juste de répertorier le terme victime sous le domaine de la catastrophe, alors qu'il pourrait appartenir aux trois autres domaines sources? Ce terme mérite d'ailleurs qu'on y revienne. La question se pose également pour les lexèmes collapse et effondrement, que nous répertorions sous le concept d'accident, alors qu'ils relèvent aussi du domaine source du bâtiment. Pour notre analyse, nous considérons l'effondrement d'une structure architecturale quelle qu'elle soit comme un accident, soit un événement imprévisible malheureux. En toute subjectivité donc, celle-ci étant inhérente au travail de taxonomie, voici les indices de conceptualisation représentatifs de chaque domaine et que nous avons cherchés dans notre corpus:

\section{LA CRISE EST UNE ÉPIDÉMIE}

epidem $^{*} /$ contagio $^{*} /$ viral / virus / immune / sick $^{*} /$ diseas $^{*} /$ ill / illness / dead / death / fatal / infect / contamina $^{*} /$ gangrene / festering / fever

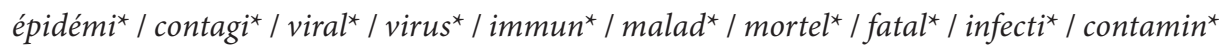
/ gangrène / purulen* / fièvre / fiévreu*

\section{LA CRISE EST UN ACCIDENT}

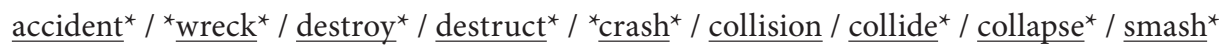
/ hit / explos / explod $^{*} /$ implo $^{*} /$ blast $^{*} /$ blow-up / blow up / detonat $^{*} /$ burst / bust / outburst / meltdown / chain reaction / flame $/$ fire / blaz $^{*}$

accident ${ }^{*}$ / naufrag* / dévast* / démoli* / détruit ${ }^{*}$ / destruct* / collision / écras ${ }^{*}$ / effondr* / fracas $^{*}$ / heurt / frapp $^{*}$ / explos I éclat $^{*}$ / implos ${ }^{*}$ / déflagration / détonation / fusion / réaction en chaîne / flamme* / feu* / incendie / brasier 


\section{LA CRISE EST UNE CATASTROPHE}

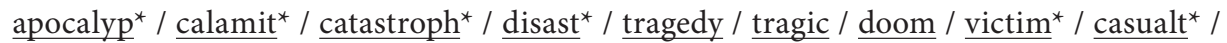
innocent bystander / sideswip $^{*} /$ hurt / damage $^{*} /$ rescue $^{*} /$ peril $^{*}$

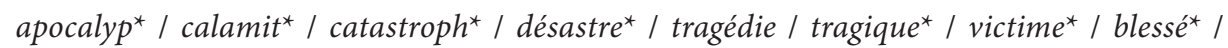
innocent ${ }^{*} /$ accroché $^{*} /$ dommage / rescousse / secour $^{*} /$ péril $^{*}$

\section{LA CRISE EST UN CATACLYSME}

cataclysm $^{*}$ / tornado / hurricane / cyclone / tsunami / wind / tidal / wave / flood / overflow / torrent $^{*} /$ waterfall / cataract / downpour / deluge / storm $^{*} /$ volcano / erupt $^{*} /$ earthquake / epicent $^{*} /$ seism $^{*} /$ shockwave $^{*} / \underline{\text { trembl}}^{*} /$ avalanche

cataclysme / tornade / ouragan / cyclone / tsunami / vent / vague / inond* / débâcle / torrent* / déluge / tempête / volcan* / éruption / tremblement de terre / épicentre / séisme* / onde* de choc / avalanche

\subsection{Résultats}

En tenant compte du nombre total de mots de chaque sous-corpus (108 500 mots anglais et 186500 mots français) par rapport aux occurrences des indices de conceptualisation, nous obtenons les résultats comparatifs suivants (tableau 2).

TABLEAU 2

Comparaison des corpus

\begin{tabular}{|c|c|c|c|c|}
\hline & $\begin{array}{l}\text { Globe and Mail } \\
\text { et Toronto Star }\end{array}$ & $\begin{array}{l}\text { Le Devoir } \\
\text { et La Presse }\end{array}$ & Mots-vedettes & Analyse et commentaire \\
\hline \multirow{2}{*}{$\frac{\text { epidemy }}{\text { épidémie }}$} & \multirow{2}{*}{0,95} & \multirow{2}{*}{1} & not immune; & \multirow{2}{*}{$\begin{array}{l}\text { Les journalistes anglophones et } \\
\text { francophones utilisent presque } \\
\text { également le concept } \\
\text { métaphorique de l'épidémie. }\end{array}$} \\
\hline & & & $\begin{array}{l}\text { contagion; } \\
\text { contaminer }\end{array}$ & \\
\hline \multirow{2}{*}{$\frac{\text { accident }}{\text { accident }}$} & \multirow[b]{2}{*}{4} & \multirow[b]{2}{*}{1} & $\begin{array}{l}\text { meltdown; is hit; } \\
\text { collapse }\end{array}$ & \multirow{2}{*}{$\begin{array}{l}\text { Les journalistes anglophones } \\
\text { utilisent quatre fois plus le concep } \\
\text { métaphorique de l'accident que les } \\
\text { journalistes francophones. }\end{array}$} \\
\hline & & & $\begin{array}{l}\text { frappé; frapper; } \\
\text { effondré; } \\
\text { effondrement }\end{array}$ & \\
\hline \multirow[b]{2}{*}{$\frac{\text { catastrophe }}{\text { catastrophe }}$} & \multirow[b]{2}{*}{1,6} & \multirow[b]{2}{*}{1} & $\begin{array}{l}\text { rescue; } \\
\text { hurt; victim }\end{array}$ & \multirow{2}{*}{$\begin{array}{l}\text { Les journalistes anglophones } \\
\text { utilisent une fois et demie plus le } \\
\text { concept métaphorique de la } \\
\text { catastrophe que les journalistes } \\
\text { francophones. }\end{array}$} \\
\hline & & & $\begin{array}{l}\text { victime; } \\
\text { catastrophe; } \\
\text { rescousse; secours }\end{array}$ & \\
\hline \multirow[b]{2}{*}{$\frac{\text { cataclysm }}{\text { cataclysme }}$} & \multirow[b]{2}{*}{0,38} & \multirow[b]{2}{*}{1} & storm & \multirow{2}{*}{$\begin{array}{l}\text { Les journalistes anglophones } \\
\text { utilisent environ trois fois moins le } \\
\text { concept métaphorique du } \\
\text { cataclysme que les journalistes } \\
\text { francophones. }\end{array}$} \\
\hline & & & $\begin{array}{l}\text { tempête; } \\
\text { débâcle; } \\
\text { onde de choc }\end{array}$ & \\
\hline
\end{tabular}

Selon les deux grandes tendances qui se dégagent, la métaphore du cataclysme prévaut dans les journaux francophones, qui l'utilisent trois fois plus que les quotidiens torontois, tandis que la métaphore de l'accident prévaut dans les journaux anglophones, qui l'utilisent quatre fois plus que les quotidiens montréalais. Parmi les indices les plus fréquents de la métaphore conceptuelle A CRISIS IS AN ACCIDENT, on retrouve meltdown, hit et collapse. Les mots tempête, débâcle et onde de choc 
reviennent le plus souvent dans le champ lexical de la métaphore LA CRISE EST UN CATACLYSME. La fréquence de ces lexèmes indique un usage systématique, de sorte qu'ils seraient devenus des cooccurrents du syntagme crise financière.

Un constat qui se pose à l'observation des domaines sources de l'accident, de la catastrophe et du cataclysme qui ont servi à créer les métaphores de la crise des subprimes est le fait qu'ils participent d'un grand paradigme: l'impondérable. Les catastrophes, les cataclysmes et les accidents ont ceci en commun qu'ils sont imprévisibles. Par transfert métaphorique, la crise est imprévisible. Loin d'être banal, le rapprochement implique un enjeu politique de taille. La croyance en l'imprévisibilité des crises financières conforte la perspective néolibérale de l'économie ${ }^{19}$, qui défend le statu quo des marchés libres et peu réglementés. Étant donné qu'il y a des honoraires considérables à gagner sur le marché des créances titrisées, dont le commerce se fait de gré à gré et n'est pas assujetti aux obligations d'information comme l'est le marché boursier, rien ne motive ceux qui s'y enrichissent à le voir réglementé par l'État. Le consensus autour de l'idée selon laquelle personne n’a vu venir ni n'aurait pu prévenir la crise convient donc stratégiquement aux parties prenantes, qui gagnent à discréditer tout effort réglementaire afin de préserver leurs intérêts (FCIC 2011: xv).

Sans faire un procès d'intention aux journalistes, il y a lieu de constater qu'ils participent à systématiser les métaphores de l'impondérable et, conséquemment, à construire un cadre cognitif fort, hors duquel il devient difficile de concevoir les mouvements du marché. Le cadre cognitif de l'impondérable, voire de force majeure ou act of God, appliqué à la crise donne à comprendre qu'il n'y a rien à faire pour prévenir celle-ci. Cette conceptualisation métaphorique participe de ce que Bourdieu nomme le "discours dominant», c'est-à-dire le produit d'une lutte économique et politique que les classes se livrent «pour imposer la définition du monde social la plus conforme à leurs intérêts» (Bourdieu 2001 : 206). Que peut-on faire pour empêcher une tempête, une débâcle, une onde de choc, ou pour prévenir la fusion au cœur d'un réacteur nucléaire (meltdown), un impact soudain (hit) ou un effondrement, autant de malheurs imprévus auxquels la crise a été comparée? Pourtant, les causes de la crise des prêts hypothécaires à risque sont imputables à l'être humain:

The crisis was the result of human action and inaction, not of Mother Nature or computer models gone haywire. The captains of finance and the public stewards of our financial system ignored warnings and failed to question, understand, and manage evolving risks within a system essential to the well-being of the American public. (FCIC 2011: xvii)

C'est peut-être davantage un problème d'agence et de représentativité qui se pose dans le discours financier journalistique. Lorsque les journalistes et les experts qu'ils citent démultiplient les métaphores de l'impondérable, ils cherchent certes à capter l'attention du lecteur et à lui faire comprendre la gravité de la situation. Or, ce faisant, ils masquent les véritables agents ${ }^{20}$ et enjeux de la crise (Negro Alousque 2010) malgré les explicitations rationnelles des causes qu'ils s'attachent à donner de celle-ci. Qui sont donc les agents financiers, qui restent innommés dans l'amalgame conceptuel «les marchés »? De manière corollaire et encore plus importante, les vraies victimes de la crise - à savoir les personnes physiques dépossédées de leur bien immobilier, de leur fonds de retraite et de leur emploi (FCIC 2011: xvi) - ne sont pas nommées. Tant dans les quotidiens francophones qu'anglophones, le mot victime désigne neuf 
fois sur dix les institutions financières faillies ou les fonds spéculatifs en difficulté parce qu'exposés aux créances hypothécaires devenues illiquides.

Loin d'être détachés, les journaux font partie intégrante des événements qu'ils observent et rapportent. En décidant du contenu de l'actualité, les journalistes insistent sur des faits que les spécialistes ne considèrent pas particulièrement pertinents et précipitent des «cascades d'attention» qui ne font qu'ajouter à la perception confuse que le public a du marché (Shiller 2000/2015: 102-103, 108). Charteris-Black va plus loin, affirmant que les journaux contribuent à semer la panique sur les marchés en assimilant les mouvements de la Bourse aux catastrophes naturelles (2004: 156). Pourtant, le travail du journaliste décrivant pour le public les informations économiques et financières est primordial parce que l'économie et la finance se trouvent souvent au cœur des débats politiques et publics (Doyle 2006: 434). Les conséquences de ce discours unifié et dominant ne sont pas des moindres dès lors que celui-ci est repris par les autorités dont on s'attendrait qu'elles cherchent à rompre l'ordre des choses, contradiction que soulignent à grands traits Catalano et Waugh:

At the same time, others involved in making policy decisions and regulations affecting the financial sector or those advocating for change and a more equitable system often use the same terms adopted by those coming from the opposite ideological frame, and thus are not able to get their message across to the public effectively $(2013: 32)$.

Un exemple éloquent du renforcement du discours dominant par ceux qui cherchent à le critiquer figure dès les premières pages du rapport où la Commission d'enquête sur la crise financière américaine livre ses conclusions: "We conclude collapsing mortgage-lending standards and the mortgage securitization pipeline lit and spread the flame of contagion and crisis" (FCIC 2011: xxiii, c'est nous qui soulignons) $)^{21}$.

\section{Conclusion}

Les métaphores économiques ont évolué du paradigme biologique au XIX ${ }^{e}$ siècle au paradigme mécaniste avec l'industrialisation (Henderson 1982). Les métaphores de l'accident et du cataclysme, en cette ère postindustrielle, pourraient inciter à croire qu'elles sont tout simplement puisées à même l'imaginaire collectif marqué par les catastrophes. Toutefois, le regard critique que la traductologie porte maintenant sur le rôle que jouent les traducteurs en tant que médiateurs culturels nous empêche de conclure sur une note aussi angélique. C'est dans une perspective sociopolitique de la traduction, définie comme "un acte délibéré et conscient de sélection, d'assemblage, de structuration et de fabrication» (Tymoczko et Gentzler 2002: XXI, notre traduction) que nous avons envisagé la production des métaphores dans le discours financier médiatique. À cet égard, la langue de spécialité est à comprendre dans le cadre plus large d'une langue structurée et structurante, dont les usagers initiés reproduisent les formes et les normes (Simeoni 2003). Qu'il s'agisse des traducteurs dans les agences de presse ou des journalistes affairés à la transédition, les rédacteurs spécialisés visent le mot juste et les expressions idiomatiques de leur domaine. L'intériorisation des formules conformes à leur technolecte - notamment les métaphores de l'impondérable devenues les cooccurrents de la langue économique et financière - résulte d'une "censure structurale», selon laquelle "c'est la structure même du champ qui régit l'expression en régissant à la fois l'accès à l'expression et 
la forme de l'expression [...]» (Bourdieu 2001 : 344). La puissance conceptuelle et la portée sociale des formules consacrées et que l'on ne questionne plus devraient être abordées dans la formation des apprentis traducteurs, futurs usagers de la langue spécialisée (Nicaise 2011 : 421). En limitant ce qui est dit, les formes imposées limitent aussi ce qui peut être perçu et pensé, et enferment le discours dans une tautologie qui devient totalité.

\section{NOTES}

1. Voir la rubrique 3.1 pour une définition.

2. Bien qu'elle figure en hapax dans l'essai phare de Smith, An Inquiry into the Nature and the Causes of the Wealth of Nations (1776), la métaphore conceptuelle «invisible hand» a servi à fonder, et sert toujours à défendre, la théorie de l'équilibre des marchés sur laquelle est basée actuellement la science de l'économie.

3. Voir la démonstration de cette machine hydromécanique, qui se trouve au musée de la Reserve Bank de la Nouvelle-Zélande: https://www.youtube.com/watch?v=FeFwyWcIHts.

4. Notamment par les travaux de Burrhus Frederic Skinner, Daniel Kahneman (prix Nobel d'économie 2002), Amos Tversky, Eldar Shafir, Ellen Langer et Robert Shiller (prix Nobel d'économie 2013), qui ont eu pour effet de montrer l'irrationalité inhérente à l'homo oeconomicus lorsqu'il se trouve confronté à des choix risqués et aux tentations du marché (Shiller 2009).

5. La sélection, dont nous avons traduit certains éléments, provient d'études qui ont observé soit le discours savant, soit le discours médiatique: Henderson 1982; 2000; Klamer et Leonard 1994; Partington 1995; Boers et Demecheleer 1997; Charteris-Black et Ennis 2001; Charteris-Black et Musolff 2003; Charteris-Black 2004; White 2004; Kermas 2006; Resche 2001; 2006; Skorczynska 2006; Rojo López et Orts Llopis 2010; Cesiri et Colaci 2011; Vasiloaia, Gaisoa-Issa, et al. 2011; Catalano et Waugh 2013. La BBC offre un palmarès ludique à http://www.bbc.com/news/business-30208476.

6. Pour toutes les occurrences répertoriées dans la liste, il s'agit d'une société de capitaux.

7. Par exemple la crise des subprimes (2007), la crise de la dette grecque (2010) et la crise de l'Eurozone (2011).

8. Par exemple «l'atome est un petit système solaire» (Gotti 2008: 58).

9. $\quad$ Nous expliquons le terme au point 4.1.

10. Le nombre des cooccurrents proposés aux rédacteurs pour combattre cette monotonie est éloquent. L'ouvrage Vocabulaire et cooccurrents de la comptabilité (Caignon 2001), sous la vedette «action", ne propose pas moins d'une trentaine de verbes pour exprimer la hausse et la baisse des cours boursiers.

11. Voir Committee on Oversight and Government Reform (2008). Greenspan a dirigé la banque centrale américaine, la Federal Reserve, de 1987 à 2006.

12. Les taux de référence sont par exemple le LIBOR et les bons du Trésor américain à six mois. Avec son jeu de mots sur l'acronyme du terme Adjustable Rate Mortgage, l'entrée "ARMed and Dangerous» de l'encyclopédie d'économie Investopedia annonce d'emblée les dangers des prêts à taux variable ou référencé.

13. Les trois institutions financières spécialisées d'intérêt public à capitaux privés qui ont participé à ce processus aux États-Unis sont Freddie Mac (Federal Home Loan Mortgage Corporation), Ginnie Mae (Government National Mortgage Association) et Fannie Mae (Federal National Mortgage Association).

14. Notamment les prêts «no-doc», «low-doc» et «NINJA» (No Income, No Job, no Assets).

15. Notre traduction du terme mortgage machine. À cette métaphore mécaniste est assortie l'idée de la chaîne de montage, qui débute par le prêt consenti à l'emprunteur et se termine par le titre souscrit par l'investisseur.

16. Il s'agit de Parvest Dynamic ABS, BNP Paribas ABS Euribor et BNP Paribas ABS Eonia.

17. «The U.S. Federal Reserve pumped about \$38-billion (U.S.) into markets yesterday in three separate interventions - its largest such operation since September of 2001. The move followed a \$24-billion injection on Tuesday. Similarly, the Bank of Canada lent out \$1.7-billion (Canadian) at low rates yesterday, mirroring its move the day before. And the European Central Bank, which started the central-bank frenzy on Tuesday with a massive \$130.7-billion (U.S.) injection, pumped about \$90-billion in yesterday.» (The Globe and Mail 11 août 2007: A1) 
18. Bien que largement utilisée en linguistique de corpus, la fréquence des unités lexicales comme révélatrice de faits pertinents pour l'analyse a fait l'objet d'une critique, notamment pour rappeler l'importance sur le plan méthodologique de considérer l'absence d'unités comme tout autant pertinente (Stefanowitsch 2006a).

19. "The current neoliberal regime [...] has reduced the range of explicit rules through the process of deregulation, partly in the belief that government regulation is the source of ambiguity, because it interferes with the market mechanism. Although advocates of the current regime embrace floating rates, derivatives, and other practices, they do so because they believe that markets are efficient and welfare-maximizing and that actors have rational expectations.» (Best 2005: 8) Le principal acquis à défendre est la loi Gramm-Leach-Bliley, adoptée en 1999 sous l'administration Clinton afin d'abolir les dernières restrictions de la loi Glass-Steagall (votée après le krach boursier de 1929), notamment l'interdiction de filiation entre les banques et les sociétés de placement.

20. Les trois membres dissidents de la commission d'enquête sur la crise financière, composée de 10 membres, imputent la crise à la contagion, aux chocs, à la panique et au grippage (FCIC 2011: 419, 432-437).

21. Les métaphores du bâtiment, de l'oléoduc, du feu et de l'épidémie s'enchevêtrent au détriment de la précision et au profit de l'effet. D’autres lexèmes du paradigme de la catastrophe (cataclys$\underline{\text { mic, }}$ catastrophe, seismic et storm) ponctuent ce rapport d'enquête long de 631 pages, mais leur fréquence n'est pas significative, soit 39 occurrences sur les 315120 mots du document. Par contre, le terme cataclysm revient dans deux passages clés qui sont les conclusions des chapitres 9 et 18, visuellement mis en évidence par un encadré et une trame grise.

\section{RÉFÉRENCES}

BAnI, Sara (2006): An analysis of press translation process. In: Kyle Conway et Susan BassnetT, dir. Translation in Global News. Coventry: University of Warwick, 35-45.

Bell, Allan (1991): The Language of News Media. Oxford: Blackwell.

Bell, Allan (1998): Approaches to Media Discourse. Oxford: Blackwell.

Best Jacqueline (2005): The Limits of Transparency. Ambiguity and the History of International Finance. Ithaca/Londres: Cornell University.

Bielsa, Esperança et BAssnett, Susan (2009): Translation in Global News. Londres/New York: Routledge.

Boers, Frank et Demecheleer, Murielle (1997): A Few Metaphorical Models in (Western) Economic Discourse. In: Gisela Redeker, Linda Waugh et Wolf-Andreas Liebert, dir. Discourse and Perspective in Cognitive Linguistics. Amsterdam/Philadelphie: John Benjamins, 115-129.

Bourdieu, Pierre (2001): Langage et pouvoir symbolique. Paris: Seuil.

Burr, Vivien (1995): An Introduction to Social Constructionism. Londres: Routledge.

Caignon, Philippe (2001): Vocabulaire et cooccurrents de la comptabilité. Montréal: Linguatech. Castells, Manuel (2000): The Information Age. Vol. 1. 2 éd. Oxford: Blackwell.

Canadian Journalism Foundation (CJF) (2011) : CJF Forum: State of Financial Journalism. Consulté le 14 octobre 2014, <http://cjffjc.podbean.com/e/state-of-financial-journalism/>.

Catalano, Theresa et Waugh, Linda R. (2013): The language of money: How verbal and visual metonymy shapes public opinion about financial events. International Journal of Language Studies. 7(2):31-60.

CESIRI, Daniela et Colaci, Laura (2011): Metaphors on the global crisis in economic discourse: A corpus-based comparison of The Economist, Der Spiegel and Il Sole 24 Ore. Rassegna Italiana di Linguistica Applicata. 1(2):201-224.

Chalmers, Patrick (2012): How to save yourself from the media's metaphors of financial mayhem. The Guardian. Consulté le 20 mars 2015, <http://www.theguardian.com/commentisfree/2012/jun/23/media-metaphors-financial-mayhem $>$.

Charaudeau, Patrick (2005/2011): Les médias et l'information. L'impossible transparence du discours. $2^{\mathrm{e}}$ éd. Bruxelles: De Boeck Université.

Charteris-Black, Jonathan (2004): Corpus Approaches to Critical Metaphor Analysis. Hampshire/New York: Palgrave Macmillan. 
Charteris-Black, Jonathan et Ennis, Timothy (2001): A comparative study of metaphor in English and Spanish financial reporting. English for Specific Purposes Journal. 20(3):249-266.

Charteris-Black, Jonathan et MusolfF, Andreas (2003): "Battered hero" or "innocent victim"? A comparative study of metaphors for euro trading in British and German financial reporting. English for Specific Purposes. 22(2):153-176.

Clark, Gordon, Thrift, Nigel et Tickell, Adam (2004): Performing Finance: The Industry, the Media and Its Image. Review of International Political Economy. 11(2):289-310.

Committee on Oversight and Government Reform (2008): The financial crisis and the role of regulators, $110^{\text {th }}$ Congress, $2^{\text {nd }}$ session. Washington: US Government Printing Office. Consulté le 20 mars 2015. <http://www.gpo.gov/fdsys/pkg/CHRG-110hhrg55764/html/ CHRG-110hhrg55764.htm>.

Conway, Kyle (2010): News translation and cultural resistance. Journal of International \& Intercultural Communication. 3(3):187-205.

DAvies, Nick (2008): Flat Earth News. Londres: Chatto et Windus.

Davis, Aeron (2002): Public Relations Democracy: Politics, Public Relations and the Mass Media in Britain. Manchester: Manchester University Press.

DAvis, Aeron (2005): Media effects and the active elite audience: A study of media in financial markets. European Journal of Communications. 20(3):303-326.

DAvis, Aeron (2006): Media effects and the question of the rational audience: lessons from the financial markets. Media, Culture \& Society. 28(4):603-625.

Dirks, Una (2006): Critical Discourse Analysis of the Iraq Conflict in the British and German 'Quality' Press. Revista Alicantina de Estudios Ingleses. 19:101-123.

DoyLe, Gillian (2006): Financial news journalism: a post-Enron analysis of approaches towards economic and financial news production in the UK. Journalism. 7(4):433-452.

Fairclough, Norman (1992): Discourse and Social Change. Cambridge: Polity Press.

FAirclough, Norman (1995): Critical Discourse Analysis. London: Longman.

FARBER, Amy (2012): Historical echoes: A water machine that simulates the economy, New York Fed Research Library, Federal Reserve Bank of New York. Consulté le 14 septembre 2013, $<$ http://libertystreeteconomics.newyorkfed.org/2012/06/historical-echoes-a-water-machinethat-simulates-the-economy.html>.

Federal CRISIS InQuiry COMmission (FCIC) (2011): The Financial Crisis Inquiry Report. Authorized Version. Consulté le 14 septembre 2013, <http://www.gpo.gov/fdsys/pkg/GPOFCIC/pdf/GPO-FCIC.pdf >.

Fowler, Roger (1985): Power. In: T. van Dijk, dir. Handbook of Discourse Analysis. Vol. 4. Londres/New York: Academic Press, 61-82.

Fowler, Roger, Hodge, Robert, Kress, Gunther, et al. (1979) : Language and Control. Londres/ Boston: Routledge/Kegan Paul.

Gambier, Yves (2006) : Transformations in International News. In: Kyle Conway et Susan BasSNETT, dir. Translation in Global News. Coventry: University of Warwick, 9-21.

Goтti, Maurizio (2008): Investigating Specialized Discourse. Bern: Peter Lang.

Haiven, Max et Berland, Jody (2014): Introduction: The financialized imagination. Topia. 30(31):7-16.

Henderson, Willie (1982): Metaphor in economics. Economics. 18(4):147-157.

Henderson, Willie (2000): Metaphor, economics and ESP: Some comments. English for Specific Purposes. 9:167-173.

JaCoBs, Geert (1999): Preformulating the News. Philadelphie: John Benjamins.

Kermas, Susan (2006): Metaphor and Ideology in Business and Economic Discourse in British and American English. In: John Flowerdew et Maurizio Gotti, dir. Studies in Specialized Discourse. Bern: Peter Lang, 109-130.

Klamer, Arjo et Leonard, Thomas G. (1994): So what's an economic metaphor? In: Philip Mirowski, dir. Natural Images in Economic Thought. Cambridge/New York: Cambridge University Press, 20-52. 
Lakoff, George (2004): Don't Think of an Elephant! White River Junction (Vermont): Chelsea Green.

LaKoff, George et Johnson, Mark (1980): Metaphors We Live By. Chicago : University of Chicago Press.

Lakoff, George et Johnson, Mark (1999): Philosophy in the Flesh. New York: Basic Books.

LALlement, Jérôme (2002) : À la recherche des objets de l'économie. In: Bernard Maris, dir. La légitimation du discours économique. Toulouse: Presses universitaires du Mirail, 9-22.

LI, Juan (2009): Intertextuality and national identity: discourse of national conflicts in daily newspapers in the United States and China. Discourse \& Society. 20(1):85-121.

LI, Juan (2010): Transitivity and lexical cohesion: Press representations of a political disaster and its actors. Journal of Pragmatics. 42:3444-3458.

Lischinsky, Alon (2011): In times of crisis: a corpus approach to the construction of the global financial crisis in annual reports. Critical Discourse Studies. 8(3):153-168.

LONGOBARDI, Elinore (2009): How 'subprime' killed 'predatory'. Columbia Journalism Review. 48(3):45-49.

MARIs, Bernard (2002): Légitimation, autolégitimation, discours expert et discours savant. In: Bernard Maris, dir. La légitimation du discours économique. Toulouse: Presses universitaires du Mirail, 109-122.

MAURIN, Bernard (2002): L'autolégitimation de la science économique par le biais de la physique. In: Bernard MARIs, dir. La légitimation du discours économique. Toulouse: Presses universitaires du Mirail, 41-54.

McCloskey, Donald N. (1983): The Rhetoric of Economics. Journal of Economic Literature. 21(2):481-517.

Molinié, Georges (1992) : Dictionnaire de la rhétorique. Paris: Librairie française générale.

Negro Alousque, Isabel (2010): The axiological value of metaphor in the business press. In: Jorge L. Bueno Alonso, dir. Analizar datos $>$ Describir variación. Analysing data $>$ Describing variation, 405-410.

NiCAise, Laurent (2011): On Going Beyond the Literal: Translating Metaphorical Conceptualizations in Financial Discourse. Meta. 56(2):407-423.

NÜNNING, Ansgar (2015): Metaphors we pay for, or: Metaphors of the 'financial crisis' shaping the cultural life of money. In: Isabel Capeloa Gil et Helena Gonçalves da Silva, dir. The Cultural Life of Money. Berlin/Boston: De Gruyter, 47-68.

Orengo, Alberto (2005): Localising news: Translation and the "global-national" dichotomy. Language and Intercultural Communication. 5(2):168-187.

Ortony, Andrew (2001): Why metaphors are necessary and not just nice. In: Martin Gannon, dir. Cultural Metaphors. Londres: Sage, 45-53.

Pander MaAt, Henk (2007): How promotional language in press releases is dealt with by journalists. Journal of Business Communication. 44:59-95.

Partington, Alan (1995): A corpus-based investigation into the use of metaphor in British business journalism. ASp. 7(10):2-12.

Partington, Alan (2006): Metaphors, motifs and similes across discourse types: CorpusAssisted Discourse Studies (CADS) at work. In: Anatol Stefanowitsch et Stefan Gries, dir. Corpus-based Approaches to Metaphor and Metonymy. Berlin/New York: Mouton de Gruyter, 267-304.

Posca, Julia (2013): Qu'est-ce que la financiarisation de l'économie. Institut de recherche et d'informations économiques. Consulté le 4 janvier 2015, <http://iris-recherche.qc.ca/blogue/ quest-ce-que-la-financiarisation-de-leconomie>.

RESCHE, Catherine (2001): Metaphors in English for economics: for a language-based approach with L2 learners. ASp. 31(3):239-259.

Resche, Catherine (2006): La métaphore dans le domaine économique: lieu d'interface entre langue et culture. In: Rosalind GreEnStein, dir. Langues et cultures: une histoire d'interface. Paris: Publications de la Sorbonne, 13-44. 
Rojo López, Ana María et Orts Llopis, María Ángeles (2010): Metaphorical pattern analysis in financial texts: Framing the crisis in positive or negative metaphorical terms. Journal of Pragmatics. 42(12):3300-3313.

SCOFFIELD, Heather (14 août 2007) : Banks intervene for third trading day. The Globe and Mail, Toronto.

ScotT, Michael (2015): WordSmith Tools version 6. Liverpool: Lexical Analysis Software.

SHILler, Robert J. (2000/2015) : Irrational Exuberance. Princeton/Oxford: Princeton University Press.

Shiller, Robert J. (2009): Human Foibles, Fraud, Manipulation, and Regulation. Open Yale Courses - Financial Markets Videos, baladodiffusion du 8 octobre 2009.

Simeoni, Daniel (2003): L'institution dans la langue: lexique et pensée d'État. TTR. 6(1):171-202.

SKorczynska, Hanna (2006): Readership and purpose in the choice of economics metaphors. Metaphor and Symbol. 21(2):87-104.

Sмiтh, Adam (1776): An Inquiry into the Nature and the Causes of the Wealth of Nations. Consulté le 14 avril 2015, <http://www.gutenberg.org/files/3300/3300-h/3300-h.htm>.

Smithers, Andrew (2013): The Road to Recovery. Chichester: Wiley.

Starkman, Dean (2009a): Power Problem. Columbia Journalism Review. 48(1):24-30.

Starkman, Dean (2009b): The list: What the business press did (and didn't do) while the financial crisis was brewing. Consulté le 14 octobre 2013, <http://www.theinvestigativefund. org/investigations/economiccrisis/1199/ the_list\%3A_what_the_business_press_did_\% 28and_didn\%E2\%80\%99t_do\%29_while_the_financial_crisis_was_brewing/>.

Stefanowitsch, Anatol (2006a): Negative evidence and the raw frequency fallacy. Corpus Linguistics and Linguistic Theory. 2(1):61-77.

Stefanowitsch, Anatol (2006b): Words and their metaphors: A corpus-based approach. In: Anatol Stefanowitsch et Stefan Gries, dir. Corpus-based Approaches to Metaphor and Metonymy. Berlin/New York: Mouton de Gruyter, 63-105.

Tambini, Damian (2010): What are financial journalists for? Journalism Studies. 11(2):158-174.

Tymoczko, Maria et Gentzler, Edwin, dir. (2002): Translation and Power. Amherst/Boston: University of Massachusetts Press.

VALDEÓn, Roberto A. (2008): Anomalous news translation. Babel. 54(4):299-326.

VANDAELE, Sylvie et Lubin, Leslie (2005): Approche cognitive de la traduction dans les langues de spécialité: vers une systématisation de la description de la conceptualisation métaphorique. Meta. 50(2):415-431.

Vandaele, Sylvie, Boudreau, Sylvie, Lubin, Leslie, et al. (2006): La conceptualisation métaphorique en biomédecine: indices de conceptualisation et réseaux lexicaux. Glottopol. Revue de sociolinguistique en ligne. 8:73-94.

Van Dijk, Teun (1988): News as Discourse. Hillsdale: Erlbaum.

VAN DijK, Teun (1995): Discourse semantics and ideology. Discourse and Society. 6(2):243-289.

VAN DijK, Teun (1998): Ideology: a Multidisciplinary Approach. Londres: Sage.

VAN Doorslaer, Luc (2010): The double text extension of translation in the journalistic field. Across Languages and Cultures. 11(2):175-188.

Vasiloaia, Mihaela, Gaisoa-Issa, Michelle et Vergara-Issa, Nora (2011): Metaphors business linguistic culture lives by. Economy Transdisciplinarity Cognition. 14(1):231-240.

White, Michael (2004): “Turbulence" and "turmoil” in the market or the language of a financial crisis. Ibérica. 7:71-86. 